\title{
Research concerning older adults with dementia
}

\author{
Adriana Cappelletti (Meds 2018) \\ Faculty Reviewers: Dr Monidipa Dasgupta, MSc, MD (Division of Geriatric Medicine), Dr Raza M Navqi, MD, FRCPC (Division of \\ Geriatric Medicine)
}

\section{BACKGROUND}

Geriatric patients are often underrepresented in clinical research, ${ }^{1}$ in part due to underrecruitment and to their often arbitrary exclusion from trials. ${ }^{2,3}$ Recruitment is especially difficult in older patients with language barriers, access barriers to leaving the home, and diseases that impair decision-making capacity., The paucity of geriatric research is troubling given Canada's aging population, ${ }^{4}$ as research findings in younger study participants may not be generalizable to older patients. ${ }^{5}$ This lack of evidence is problematic for health care providers, who must decide whether prescribing certain treatments to older patients is appropriate without the proper evidence base. ${ }^{1}$

Unique ethical and legal challenges exist surrounding recruitment of incapacitated older patients, specifically within the context of dementia. Between $44 \%$ and $67 \%$ of patients with mild-to-moderate dementia do not have the decision-making ability to consent to participating in research, and all patients with severe dementia are considered incapacitated. ${ }^{6}$ However, their inclusion in research is essential to furthering investigators' understanding of dementia. For example, both cerebrovascular and cardiovascular disease (CVD) have been associated with cognitive decline; without the participation of patients with late-stage dementia, researchers cannot thoroughly investigate how CVD influences the progression of cognitive decline or, contrarily, how dementia may put a patient at risk of CVD. ${ }^{7}$ This article explores how older adults with dementia may be included in clinical research while protecting these older adults from coercion or harm.

\section{LEGAL STANDARDS FOR DECISION-MAKING}

In considering the legal challenges of geriatric research in incapacitated older populations, it is important to first define capacity. Although not a universal definition, the "four abilities model" devised by Appelbaum and Grisso describes four criteria to define legal standards of capacity: being able to reason about options, communicating a decision, understanding the context of that decision and appreciating its foreseeable consequences. ${ }^{6,8,9}$ Many patients with dementia do not meet these standards of capacity and are thus unable to provide consent themselves when participating in research. ${ }^{6,10}$ In Canada, the Tri-Council Policy Statement (TCPS) on Ethical Conduct for Research Involving Humans dictates that only a substitute decision-maker (SDM) who is not part of the research team may authorize an incapacitated adult's participation in research. ${ }^{11}$ Although the SDM's consent is required for participation, the patient's dissent (ie objection) to participation overrules the SDM's decision. ${ }^{11,12}$ Therefore, investigators should provide incapacitated patients with as much information about the trial as patients' understanding will allow, in an effort to obtain their affirmative agreement, otherwise known as their assent. ${ }^{13}$ In the context of dementia, assent is defined as the ability to express a choice and at least a minimal understanding of the information provided. The inability to communicate a choice and thereby provide assent does not preclude a patient with dementia from participating in research; however, assent is required for patients who have this ability, and dissent to participation is unequivocal. ${ }^{12}$

The TCPS also states that incapacitated adults may only be recruited for research specifically requiring their participation (eg an Alzheimer's disease treatment study) and involving either minimal risk or risk that is outweighed by potential direct benefits. ${ }^{11}$ These standards parallel the protective measures in place for paediatric research. ${ }^{14}$ There is no clear threshold of when potential risks outweigh direct or indirect benefits. Thus, to balance scientific inquiry with protection of patients, review of protocols for research of incapacitated older adults must consider broad risk-benefit categories, and protocols with no anticipated benefits to study participants require more conservative risk-benefit analyses..$^{10}$

\section{ETHICAL CONSIDERATIONS}

The decision to include an older, incapacitated participant in research must respect that individual's values and address principles of beneficence, non-maleficence and justice. ${ }^{15,16}$ There is disagreement in the literature regarding the most ethical approach to making this decision. Two commonly considered approaches are "best interests" and "substituted judgments". In substituted judgments, the SDM makes a decision based on what the participant may have believed, thereby aiming to respect the participant's values and wishes. A concern with substituted judgments is that often an SDM may be unaware of the patient's prior wishes since consent to research is not routinely incorporated in a patient's advance directives. ${ }^{17}$

In contrast to substituted judgments, which emphasize the patient's prior wishes, "best interests" values the SDM's opinion on what is best for the patient. "Best interests" aims to maintain the older adult's quality of life and avoid unnecessary harm, should the intervention pose risks. ${ }^{17}$ Making this judgment is challenging in the case of established treatments and becomes even more so when evaluating an experimental treatment with unknown risks and benefits. Another concern with this more paternalistic approach is the possibility of the older adult's previous wishes being disrespected.

With either approach, it is very difficult to determine whether a person without the ability to consent would be interested in therapies with potential risks that may prolong life without improving quality of life..$^{15-17}$ However, the potential benefits of this research to 
patients with dementia cannot be uncovered unless resources are justly allocated towards studies involving this population of incapacitated older adults.

\section{FUTURE DIRECTIONS AND CONCLUDING REMARKS}

In the context of research involving older, incapacitated adults, be it with dementia or other mentally impairing diseases, the best course for recruiting patients is not clear. Inclusion of incapacitated participants is essential to enhancing our understanding of dementia and might have direct neurological benefits on these individuals. ${ }^{6,10}$ However, SDMs face the challenge of weighing the risks and benefits of clinical research to their relative, often having to speculate what their relative's wishes would be. ${ }^{17}$ To address this concern, interest in research trials should be discussed with individuals with early dementia while they are still able to decide for themselves.

Involvement of incapacitated older adults in research trials may also be improved by training research personnel on evaluating capacity, ${ }^{6}$ by avoiding arbitrary upper age limits in protocols and by addressing access and transport requirements of older adults. ${ }^{2}$ Additionally, expanding academic geriatrics and involving a greater number of geriatricians on research boards would help to promote geriatric research overall. ${ }^{1}$

\section{REFERENCES}

1. Ilgili O, Arda B, Munir K. Ethics in geriatric medicine research. Turk Geriatr Derg. 2014;17(2):188-95.

2. McMurdo MET, Roberts H, Parker S et al. Improving recruitment of older people to research through good practice. Age and Ageing. 2011;40(6):659-65.

3. Dunn LB, Fischer SR, Hantke M et al. "Thinking about it for somebody else": Alzheimer's disease research and proxy decision makers' translation of ethical principles into practice. Am J Geriatr Psychiatry. 2013 April;21(4):337-45.

4. Indicators of Well-being in Canada [Internet]. Canada; Employment and Social Development Canada; Canadians in context-aging population. 2015 Apr 18 [cited 2015 Apr 19]. Available from: http://www4. hrsdc.gc.ca/.3ndic.1t.4r@-eng.jsp?iid=33.

5. O'Hare AM, Hotchkiss JR, Tamura MK et al. Interpreting treatment effects from clinical trials in the context of real-world risk information: end-stage renal disease prevention in older adults. JAMA Intern Med. 2014;174(3):391-7.

6. Kim SYH, Karlawish JHT, Eric D. Current state of research on decision-making competence of cognitively impaired elderly persons. Am J Geriatr Psychiatry. 2002;10(2):151-65.

7. Qui C, Fratiglioni L. A major role for cardiovascular burden in age-related cognitive decline. Nat Rev Cardiol. 2015 May;12(5):267-77.

8. Appelbaum PS. Assessment of patients' competence to consent to treatment. New Engl J Med. 2007;357:1834-40.

9. Grisso T, Appelbaum PS. Assessing competence to consent to treatment: a guide for physicians and other health professionals. New York: Oxford University Press, 1998.

10. Kim SYH, Appelbaum PS, Jeste DV, Olin JR. Proxy and surrogate consent in geriatric neuropsychiatric research: update and recommendations. Am J Psychiatry. 2004;161:797-806.
11. Canadian Institutes of Health Research, Natural Sciences and Engineering Research Council of Canada, and Social Sciences and Humanities Research Council of Canada. Tri-council policy statement: ethical conduct for research involving humans [Internet]. Canada: Government of Canada; 2014 Dec [cited 2015 May 25]. Available from: http:// www.pre.ethics.gc.ca/pdf/eng/tcps2-2014/TCPS_2_FINAL_Web.pdf.

12. Black BS, Rabins PV, Sugarman J, Karlawish JH. Seeking assent and respecting dissent in dementia research. Am J Geriatr Psychiatry. 2010 Jan;18(1):77-85.

13. International Conference on Harmonisation of Technical Requirements for Registration of Pharmaceuticals for Human Use: Guideline on good clinical practice: consolidated guideline [Internet]. Canada: Health Canada; 1997 [updated 2004 Feb 2; cited 2015 May 26]. Available from: http://www.hc-sc.gc.ca/dhp-mps/alt_formats/hpfb-dgpsa/ pdf/prodpharma/e6-eng.pdf.

14. McRae AD. Research ethics [Internet]. Royal College of Physicians and Surgeons of Canada; 2014 [cited 2015 Mar 22]. Available from: http:// www.royalcollege.ca/portal/page/portal/rc/resources/bioethics/ primers/research_ethics\#adults.

15. Dunn LB, Misra S. Research ethics issues in geriatric psychiatry. Psychiatr Clin North Am. 2009 Jun;32(2):395-411.

16. Bravo G, Paquet M, Dubois MF. Knowledge of the legislation governing proxy consent to treatment and research. J Med Ethics. 2003;29:44-50.

17. Berger JR. Is best interests a relevant decision making standard for enrolling non-capacitated subjects into clinical research? J Med Ethics. 2011;37:45-9. 